\title{
Seismic Data Matching by least-squares non-stationary triangle smoothing
}

Reem Alomar and Sergey Fomel, The University of Texas at Austin

Copyright 2021, SBGf - Sociedade Brasileira de Geofísica

This paper was prepared for presentation during the $17^{\text {th }}$ International Congress of the Brazilian Geophysical Society held in Rio de Janeiro, Brazil, 16-19 August 2021.

Contents of this paper were reviewed by the Technical Committee of the $17^{\text {th }}$ International Congress of the Brazilian Geophysical Society and do not necessarily represent any position of the SBGf, its officers or members. Electronic reproduction or storage of any part of this paper for commercial purposes without the written consent of the Brazilian Geophysical Society is prohibited.

\section{Abstract}

We propose a fast and accurate method to estimate the non-stationary triangle smoothing radius for matching seismic datasets. The smoothing radius is estimated by non-linear least-squares inversion using an iterative Gauss-Newton approach. We derive and implement the derivative of the smoothing operator to compute the gradient for inversion. The efficiency of the proposed method is confirmed in two applications: least-squares migration and matching low-resolution and high-resolution seismic images from the same exploration areas.

\section{Introduction}

Triangle smoothing is a widely used and efficient filtering operation that finds application in regularizing seismic inverse problems and computing local attributes (Fomel, 2007a; 2007b).

Greer and Fomel (2018) developed an iterative method for an iterative estimation of the smoothing radius for nonstationary smoothing in matching two seismic datasets. The method is based on the local frequency attribute and has been applied successfully for approximating the inverse Hessian operator in least-squares migration (Greer et al., 2018).

Chen (2021) introduced a multi-dimensional non-stationary triangle smoothing operator in local time-frequency transformation (Liu and Fomel, 2013) to address the nonstationary nature of the input seismic data. This transformation was proven to be useful in several practical applications of time-frequency analysis. Chen and Fomel (2021) proposed a new non-stationary local signal and noise orthogonalization method as an alternative to local signal-and-noise orthogonalization method (Chen and Fomel, 2015). In this approach, the stationary triangle smoothing constraint for the local orthogonalization weight becomes a non-stationary smoothing constraint. For highly non-stationary data, the smoothing radius is small where the signal is dominant, and it is large where the noise is dominant; thus, the radius adapts to achieve the optimal stability and accuracy. Wang et al. (2021) proposed a robust non-stationary local slope estimation method that balances both the stability and the resolution of slope perturbations by controlling the strength of triangle smoothing in the shaping regularization framework within the plane-wave destruction algorithm (Fomel, 2002).
While these new non-stationary methods improve resolution and accuracy, they also introduce additional computational cost with the necessary step of estimating the non-stationary triangle smoothing radius. Chen and Fomel (2021) noted that the non-stationary local orthogonalization method compared to the stationary method is almost 15 times slower due to the timeconsuming step of estimating the non-stationary triangle smoothing radius. While the iterative method of Greer and Fomel (2018) is robust and effective, it does not provide an optimally fast convergence.

In this paper, we propose an alternative method to estimate the non-stationary triangle smoothing radius. We derive a new operator - the triangle smoothing derivative, which is used to guide better estimates for the radius in regularized least-squares inversion using an iterative Gauss-Newton approach.

\section{Triangle Smoothing}

A box filter is defined in the Z-transform notation as follows (Claerbout, 1992):

$$
\begin{gathered}
B(Z)=\frac{1}{N}\left(1+Z+Z^{2}+\cdots+Z^{N-1}\right)=\frac{1}{N} \frac{\left(1-Z^{N}\right)}{(1-\mathrm{Z})} \\
Z=e^{i w \Delta t}=\cos (w \Delta t)+i \sin (w \Delta t)
\end{gathered}
$$

where $N$ is the number of samples included in a moving average under a rectangular window, $w$ is the frequency in radians, and $\Delta t$ is the interval spacing in time. Division by $(1-\mathrm{Z})$ is the operation of causal integration and it corresponds to the following recursion in time:

$$
y_{n}=x_{n}-x_{n-1}
$$

The adjoint of this operation is anti-causal integration, or the division by $\left(1-Z^{-1}\right)$, and is represented by the following recursion:

$$
x_{n}=y_{n}+x_{n-1}
$$

A triangle filter is defined as the cross-correlation of two box filters (Claerbout, 1992):

$$
T(Z)=B(Z) B\left(Z^{-1}\right)=\frac{1}{N^{2}}\left(\frac{\left(2-Z^{N}-Z^{-N}\right)}{(1-Z)\left(1-Z^{-1}\right)}\right.
$$

where $N$ is the triangle smoothing radius, or half the number of points averaged under a triangle window. Triangle smoothing is efficient because it requires only $n$ multiplications for $n$ data samples followed by a chain of causal and anti-causal integration independent of the size of the smoothing radius. By expanding $Z$ and redefining the triangle smoothing radius as $R$, we can define a triangle filter as a function of the smoothing radius and frequency:

$$
T(R, w)=\frac{1}{R^{2}}\left[\frac{2-2 \cos (R w \Delta t)}{2-2 \cos (w \Delta t)}\right]=\frac{1}{R^{2}}\left[\frac{\sin \left(\frac{R w \Delta t}{2}\right)}{\sin \left(\frac{w \Delta t}{2}\right)}\right]^{2}
$$


To implement smoothing with a non-integer radius $R$ in the time domain, we interpolate the results of smoothing by the integer radius $N$ and $N+1$. After choosing the appropriate weighting coefficients, we define the approximate triangle smoothing function for a non-integer smoothing radius as the following:

$$
T_{\text {non-integer }}(R, w)=\left[\frac{(N+1)^{2}-R^{2}}{(N+1)^{2}-N^{2}}\right] T(N, w)+\left[\frac{R^{2}-N^{2}}{(N+1)^{2}-N^{2}}\right] T(N+1, w)
$$

To justify the weighting coefficients we chose, we match the $2^{\text {nd }}$ order Taylor expansion of equations (6) and (7) around the zero frequency $w=0$ :

$$
T(R, w=0)=T_{\text {non-integer }}(R, w=0) \approx \frac{-1}{12}\left(R^{2}-1\right) w^{2}+1
$$

The approximations match to the second order, similar to the accuracy of triangle smoothing in approximating the ideal Gaussian smoother.

\section{Triangle Smoothing Derivative}

We now proceed with introducing a new operator, the triangle smoothing derivative, which is obtained by taking the derivative of equation (6) with respect to the radius $R$ :

$$
\frac{d T}{d R}(R, w)=[i w]\left[\frac{-i \Delta t \sin \left(\frac{R w \Delta t}{2}\right)}{2 R^{2} \sin ^{2}\left(\frac{w \Delta t}{2}\right)}\right]-\frac{2}{R} T(R, w)
$$

We implement the triangle smoothing derivative in the time domain using the following three steps:

1) A digital filter analogous to smoothing involving a chain of causal and anti-causal integration.

$$
F(Z)=\frac{1}{N^{2}} \frac{Z^{N}-Z^{-N}}{(1-Z)\left(1-Z^{-1}\right)}
$$

2) Approximating the derivative of the result of step 1 by taking the $2^{\text {nd }}$ order central difference.

3) Subtracting from the result of step 2 the result of smoothing normalized by $\frac{2}{R}$.

To approximate the triangle smoothing derivative function for a non-integer radius we use the following interpolation:

$$
\begin{array}{r}
\frac{d T}{d R}{ }_{\text {non-integer }}(R, w)=[(N+1)-R] \frac{d T}{d R}(N, w)+ \\
{[R-N] \frac{d T}{d R}(N+1, w)}
\end{array}
$$

The weighting coefficients we chose here are justified by matching the $2^{\text {nd }}$ order Taylor expansion of equations (9) and (11) around the zero frequency $w=0$ :

$$
\frac{d T}{d R}(R, w=0)=\frac{d T}{d R}_{\text {non-integer }}(R, w=0) \approx \frac{-1}{6} R w^{2}
$$

Both triangle smoothing and the triangle smoothing derivative have a straightforward non-stationary implementation where the smoothing radius changes for each point along a trace. The non-stationary implementation is a direct extension of the stationary implementation because all equations depend directly on the radius.

\section{Estimating the Smoothing Radius}

Now that we have obtained the triangle smoothing derivative, we can develop a method to estimate the radius given the original and filtered datasets. We utilize the Gauss-Newton approach of solving a non-linear leastsquares problem (Lawson and Hanson, 1995). We define a triangle smoothing operator with radius $R$ applied to data $d$ as $S_{R}[d]$, and a triangle smoothing derivative operator as $S_{R}^{\prime}[d]$. Given the original data $d_{\text {input }}$, we define the following Taylor expansion:

$$
S_{R}\left[d_{\text {input }}\right] \approx S_{R_{0}}\left[d_{\text {input }}\right]+S_{R_{0}}^{\prime}\left[d_{\text {input }}\right]\left(R-R_{0}\right)
$$

where $R$ is the best estimate for the radius and $R_{0}$ is the first guess for the radius. Noting that $S_{R}\left[d_{\text {input }}\right]=d_{\text {output }}$, the filtered data, we rearrange equation (13) to solve for $R$ :

$$
R \approx R_{0}+\frac{d_{\text {output }}-S_{R_{0}}\left[d_{\text {input }}\right]}{S_{R_{0}}^{\prime}\left[d_{\text {input }}\right]}
$$

We can repeat this approach and solve for the radius iteratively, where the radius at the $i^{\text {th }}$ iteration is given by:

$$
R_{i+1}=R_{i}+\frac{d_{\text {output }}-S_{R_{i}}\left[d_{\text {input }}\right]}{S_{R_{i}}^{\prime}\left[d_{\text {input }}\right]}
$$

This method in theory converges in a rate approaching quadratic, although the convergence is not guaranteed if the initial guess is far from the true value (Lawson and Hanson, 1995).

The proposed method is directly extended to solve for a non-stationary triangle smoothing radius given that the triangle smoothing derivative is non-stationary as well. Defining the non-stationary triangle smoothing operator as $n S$ and the non-stationary triangle smoothing derivative operator as $n S^{\prime}$, we can estimate the non-stationary radius $n R_{i}$ at the $i^{\text {th }}$ iteration as follows:

$$
n R_{i+1}=n R_{i}+\frac{d_{\text {output }}-n S_{n R_{i}}\left[d_{\text {input }}\right]}{n S_{n R_{i}}^{\prime}\left[d_{\text {input }}\right]}
$$

Note that for stability of the solution, we must take care in performing the division in equation (16). We implement smooth division which treats division as inversion and regularizes the inversion using shaping regularization (Fomel, 2007a). The shaping regularization is controlled by its own smoothness radius and corresponds to stationary least-square estimate when the smoothing radius for shaping is set to be very large.

\section{First application: Least-squares migration}

Greer et al. (2018) proposed a method to improve migration resolution by approximating the least-squares Hessian operator using non-stationary amplitude and frequency matching between the first and second conventionally migrated images. Following Greer et al. (2018), we perform this application on a 2-D synthetic dataset created by Claerbout (2006) using Kirchhoff poststack migration and highlight the improvements in resolution and computational cost by using the new method to estimate the triangle smoothing radius. 
The frequency matching step is performed by estimating the non-stationary triangle smoothing radius to match local frequencies between the first and second conventionally migrated images. We perform both the line-search method (Greer and Fomel, 2018) and the proposed Gauss-Newton approach to estimate the non-stationary triangle smoothing radius. Our proposed method compared with the linesearch method produces a more smoothly varying radius, as seen in Figure 1. This is a desired result because a highly variable radius produces an image with highfrequency noise that must be mitigated with regularization or bandpass filtering (Figure $2 \mathrm{~B}$ ). The image we produce with the proposed method to estimate the radius better matches the starting model without a need for smooth regularization (Figure $2 \mathrm{C}$ ).

Further, these images can be used as better starting models for least-squares migration (LSM). Figure 3 shows the errors in iterative LSM comparing standard LSM, starting model provided by line-search method, and starting model provided by Gauss-Newton method. The proposed method provides the best starting model for LSM.

A)

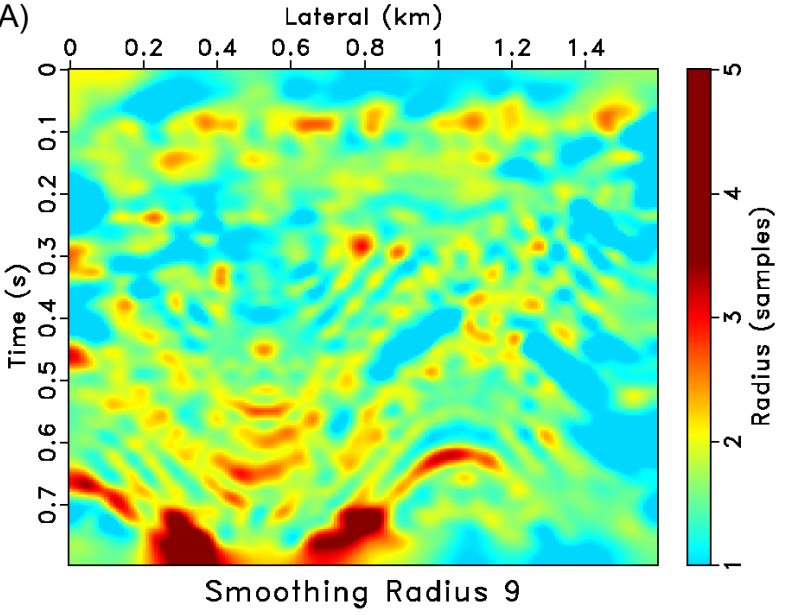

B)



Smoothing Radius 9

Figure 1 - Estimated triangle smoothing radius after 9 iterations for A) line-search method and B) Gauss-Newton method.
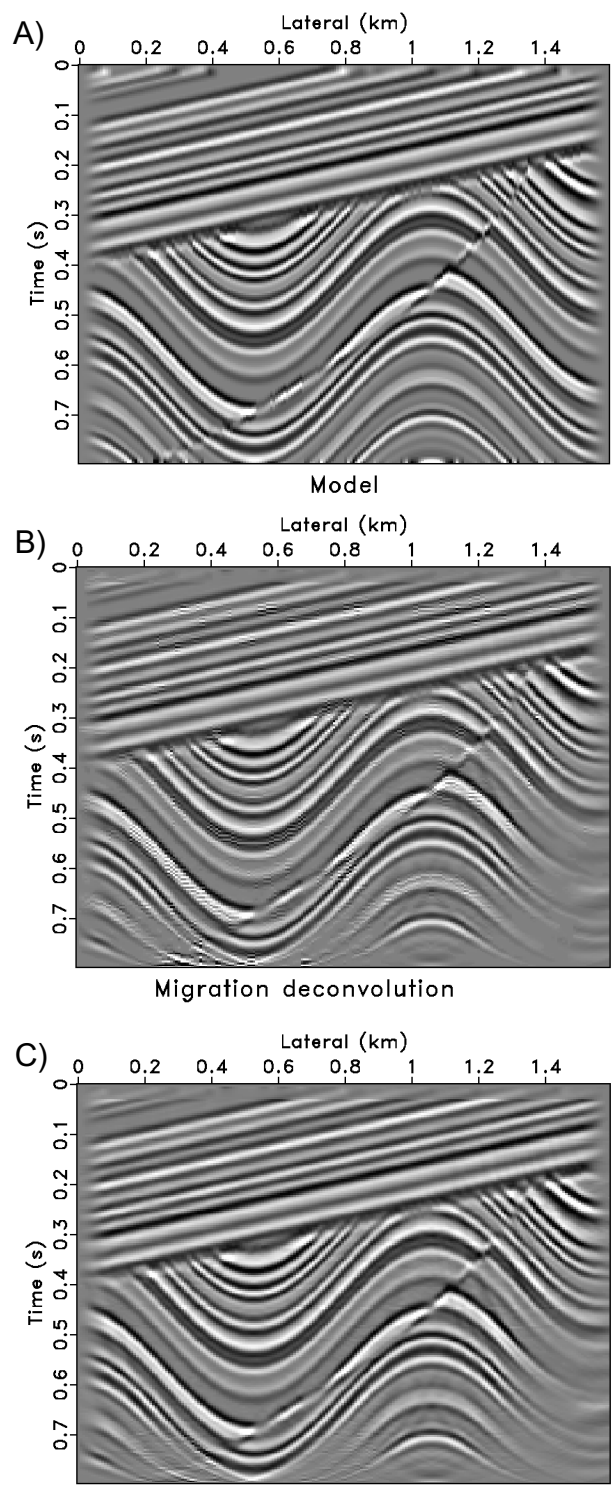

Migration deconvolution

Figure 2 - A) 2-D synthetic reflectivity model (Claerbout, 2006) B) Migration deconvolution line-search approach C) Migration deconvolution Gauss-Newton approach

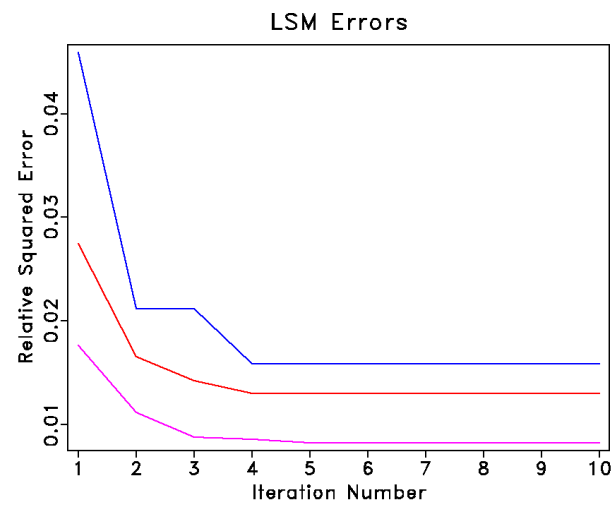

Figure 3 - Least-squares migration (LSM) errors for standard LSM (blue), LSM with starting model from line-search approach (red), and LSM with starting model from GaussNewton approach (magenta) 


\section{Second Application: Matching legacy and high- resolution seismic images}

We apply our new method to estimate the radius in the workflow of matching and merging high-resolution seismic data and legacy data that were acquired over the same area (Greer and Fomel, 2018). The high-resolution seismic image has a larger frequency bandwidth and a higher dominant frequency, producing a high-resolution image of the shallow subsurface (Figure 4A). Legacy image, on the other hand, contains important low-frequency content, thus producing better depth coverage (Figure 4B).

The first step in matching and merging the two images is to match their spectral content. We initially apply a highpass filter to the legacy data to remove the low frequencies that are not present in the high-resolution data. We then smooth the high-resolution image with a non-stationary triangle smoothing radius to match its local frequency content with the legacy image. The non-stationary triangle smoothing radius is estimated by directly implementing the algorithm in equation (20) by substituting the highresolution image as the input data $d_{\text {input }}$, and the legacy image as the filtered data $d_{\text {output }}$. The starting model for the radius is chosen carefully to preserve stability. The initial guess for the radius shown in figure $6 \mathrm{~A}$ is a smoothed version of the theoretical smoothing radius proposed by Greer and Fomel (2018). The estimated radius after 7 iterations is shown in figure 6B. We compare both the spectral content (Figure 7) and the local frequency content (Figure 8) of the images before and after smoothing. The frequency content is better balanced after smoothing with the newly estimated radius.
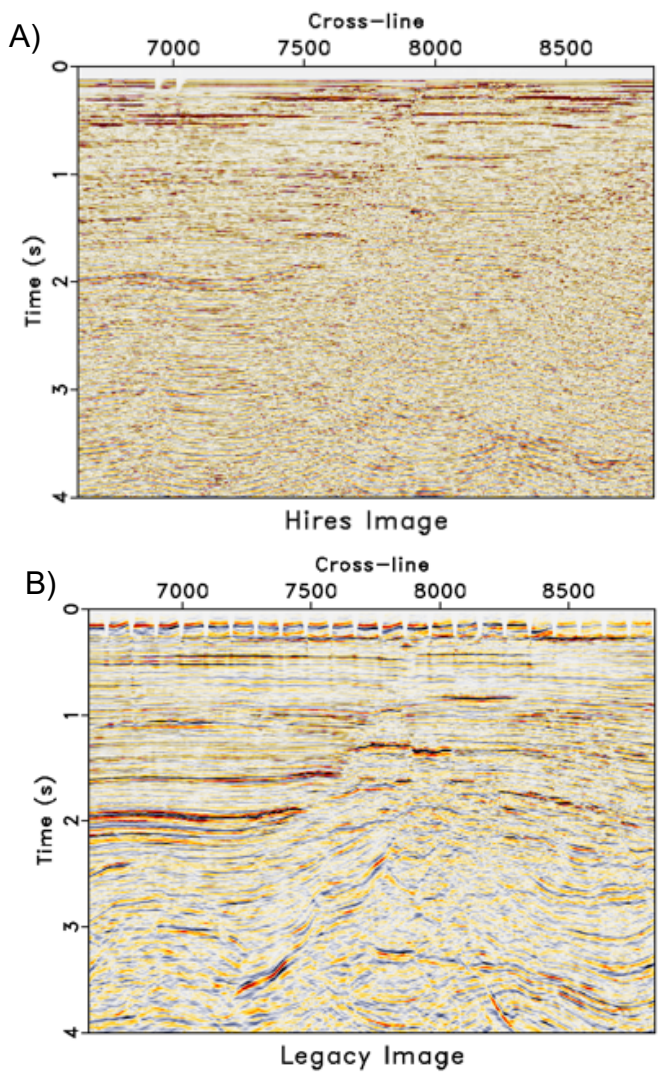

Figure $4-A)$ High-resolution Image and B) Legacy Image
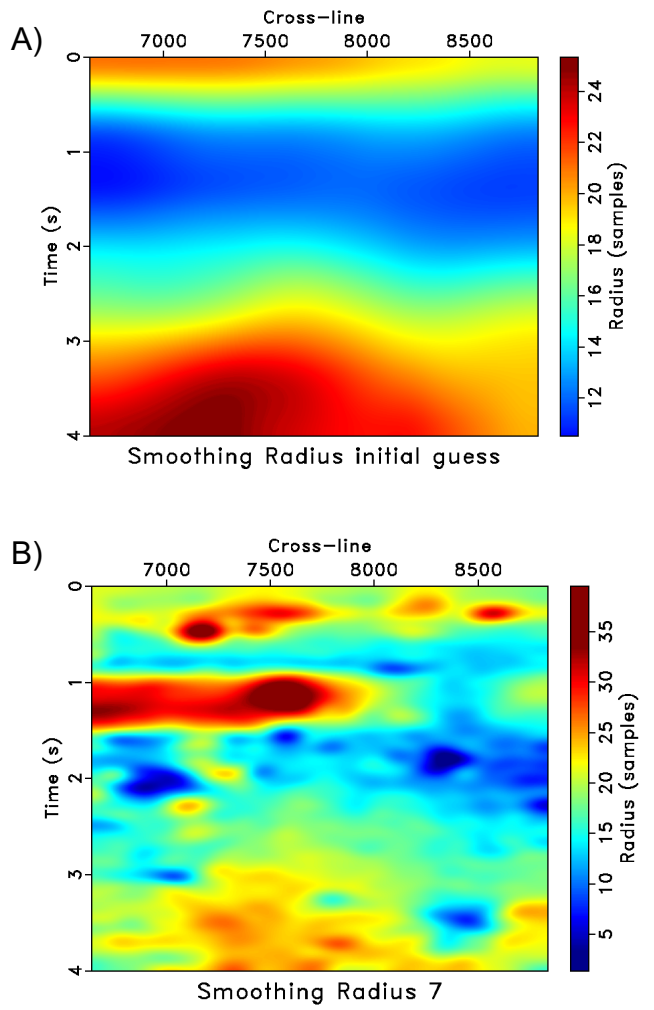

Figure 5 - Initial guess for the smoothing radius ( $A$ ) and estimated smoothing radius after 7 iterations $(B)$.

A)

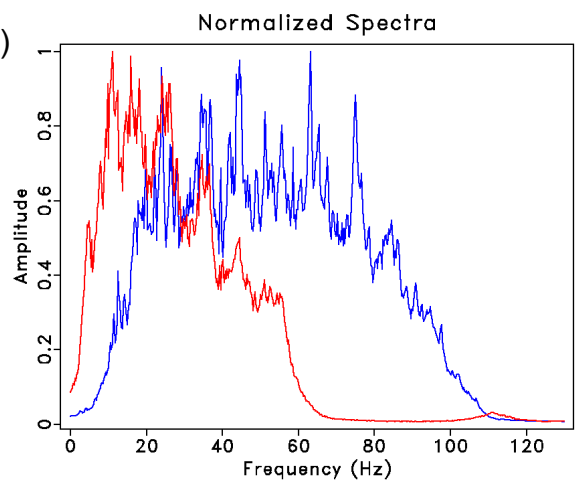

B)

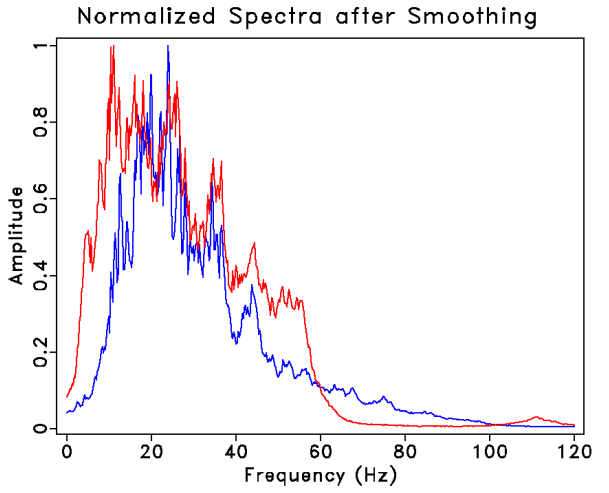

Figure 6 - Normalized spectra of legacy (red) and highresolution (blue) before $(A)$ and after $(B)$ non-stationary smoothing.

Seventeenth International Congress of the Brazilian Geophysical Society 


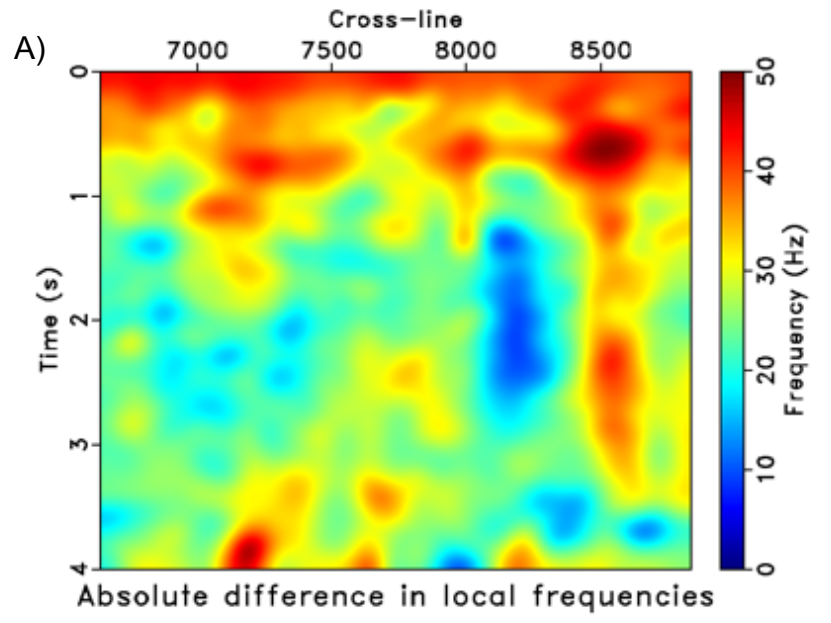

B)

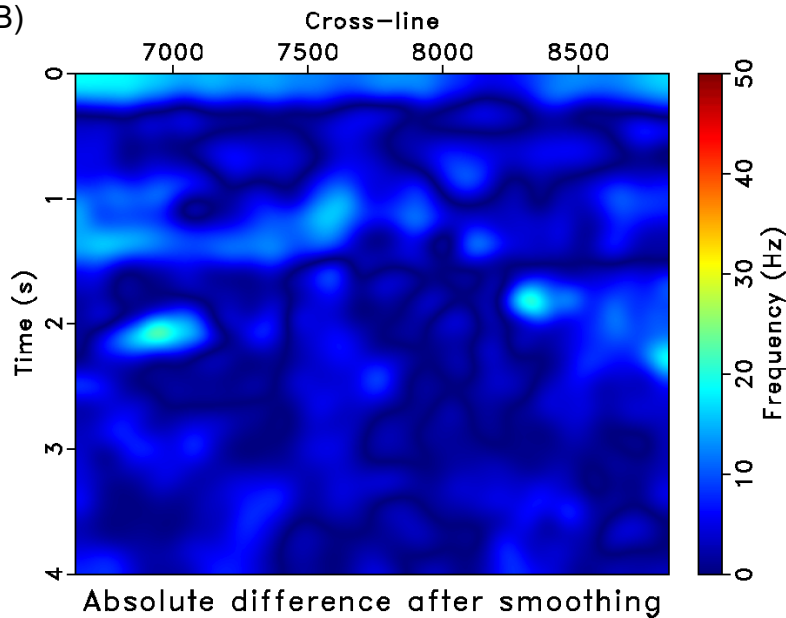

Figure 7 Absolute difference in local frequencies between Legacy and high-resolution before $(A)$ and after $(B)$ nonstationary smoothing of the high-resolution data.

\section{Conclusions}

We have introduced a fast and accurate method to estimate the non-stationary triangle smoothing radius for matching seismic datasets. We derive and implement a non-stationary triangle smoothing derivative operator that guides better guesses for the radius in regularized leastsquares inversion using an iterative Gauss-Newton approach. Our method converges with a fast quadratic rate of convergence given an appropriate starting model for the radius. This method was shown to be effective in applications to improving migration resolution and to matching legacy and high-resolution seismic images. It can find additional application in other geophysical data analysis tasks.

\section{Acknowledgments}

We thank Yangkang Chen and Sarah Greer for inspiring discussions and sponsors of the Texas Consortium for Computational Seismology (TCCS) for their financial support.

\section{References}

Chen, Y., 2021. Nonstationary local time-frequency transform. GEOPHYSICS, 86(3), pp.V245-V254.

Chen, Y. and Fomel, S., 2015. Random noise attenuation using local signal-and-noise orthogonalization. GEOPHYSICS, 80(6), pp.WD1-WD9.

Chen, Y. and Fomel, S., 2021. Non-stationary local signaland-noise orthogonalization. GEOPHYSICS, accepted.

Claerbout, J. F., 1992, Earth Soundings Analysis: Processing Versus Inversion: Blackwell Scientific Publications.

Claerbout, J. F., 2006. Basic Earth Imaging: Stanford Exploration Project.

Fomel, S., 2002. Applications of plane-wave destruction filters. GEOPHYSICS 67(6), pp.1946-1960.

Fomel, S., 2007a. Shaping regularization in geophysicalestimation problems. GEOPHYSICS, 72(2), pp.R29-R36.

Fomel, S., 2007b. Local seismic attributes. GEOPHYSICS, 72(3), pp.A29-A33.

Greer, S. and Fomel, S., 2018. Matching and merging high-resolution and legacy seismic images. GEOPHYSICS, 83(2), pp.V115-V122.

Greer, S., Xue, Z. and Fomel, S., 2018. Improving migration resolution by approximating the least-squares Hessian using nonstationary amplitude and frequency matching. In SEG Technical Program Expanded Abstracts 2018, pp. 4261-4265.

Lawson, C. L., and Hanson, R. J, 1995. Solving Least Squares Problems. SIAM.

Liu, Y. and Fomel, S., 2013. Seismic data analysis using local time-frequency decomposition. Geophysical Prospecting, 61(3), pp.516-525.

Wang, H., Huang, G. and Chen, Y., 2021. Robust Nonstationary Local Slope Estimation. IEEE Transactions on Geoscience and Remote Sensing, accepted. 